\title{
Testimonio: Pedro Ortiz Cabanillas (1933-2011) Testimony: Pedro Ortiz Cabanillas (1933-2011)
}

\author{
Alberto Perales C. \\ Profesor de Psiquiatría. Ex Director del Instituto de Ética en Salud de la Facultad de Medicina, Universidad Nacional Mayor de San Marcos. \\ Doctor en Medicina. Vice-Presidente, Academia Nacional de Medicina.
}

An Fac med. 2011;72(1):83-8

En el curso de mi ya 'no corta existencia', expresión que prefiero a la de 'mi larga existencia', he conocido personalidades -en el concepto que Ortiz propone en su teoría sociobiológica informacional del desarrollo moral del Hombre- que, a diferencia de muchos otros, parecieran saber exactamente por dónde caminar en este mundo. Pareciera, digo, por la imagen que transmiten, de conocer su misión terrenal o de ser guiados por una extraña fuerza conductora hacia objetivos trascendentes. Vale decir, siguen el trazo o la ruta de un destino.

El significado de este término, destino, sencillo en apariencia, es finalmente de complejo análisis. Joan Corominas nos informa que el vocablo es una derivación acuñada en el S. XVI, de la voz destinar, del latín destinare, que propiamente significa 'fijar, sujetar', 'apuntar, hacer puntería (hacia).

Recordemos que desde el fatum, aquello que predecía el oráculo, el destino constituye un concepto que expresa la noción religiosa e idealista de una fuerza sobrenatural que predetermina todos los acontecimientos en la vida de las personas. Según la mitología griega, la suerte de las personas, e incluso de los dioses, dependía de las deidades del destino. A estas diosas los griegos las llamaban moiras, mientras que los romanos las denominaban parcas.

Spengler señalaba que la idea del destino es radicalmente opuesta a la del principio de causalidad. El primero se refiere al Universo - Historia, cuya comprensión es intuitiva, mientras que el segundo al Universo-Naturaleza, cuya comprensión es lógica y racional. El destino se asocia a la vida, la causalidad a la muerte.

En torno a estas reflexiones trataré de describir la personalidad de quien fuera Pedro Ortiz Cabanillas, pues siempre me pregunté qué moiras o qué parcas iluminaban su brillante camino $^{(*)}$.

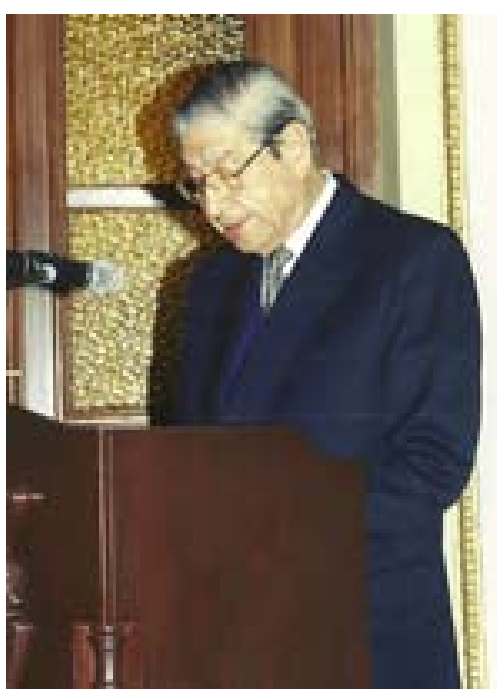

Figura 1. Dr. Pedro Ortiz Cabanillas, dando su discurso de asunción al cargo de Director del Instituto de Ética en Salud.

* El artículo se basa, en parte, en el discurso de presentación pronunciado por el autor, con ocasión de las Palmas

Magisteriales que recibiera el Dr. Ortiz en el año 2006. 


\section{SU VIDA}

Pedro nació un 31 de julio de 1933, en Cajamarca, en la linda ciudad de Celendín. Su vida, que a juzgar por su circunstancia podría haberlo conducido, como a tantos otros peruanos, por sendas mucho más modestas, floreció, por el contrario, resilientemente ante las dificultades, a base de un entorno familiar y social solidario y generoso, y por algo más que solo puedo llamar destino. Este, sin explicación lógica posible, apareció en momentos de decisiones importantes, ayudándolo a sortear los muchos inconvenientes que la vida le planteara.

En una de nuestras tantas tertulias me contó que su padre, Arturo Ortiz Sánchez, había fallecido de una complicación quirúrgica, cuando él tenía alrededor de 4 años. Su madre debió viajar a Lima, para regentar un colegio y aliviar la presión del presupuesto familiar. Su único hermano, aún muy pequeño, fue el obligado compañero de viaje de aquella, debiendo Pedro permanecer en Celendín. Aquí, podríamos decir, se observa la primera señal de su destino, porque en casos similares, la experiencia clínica psiquiátrica y las teorías psicológicas enseñan que la ausencia de los padres, durante los primeros años de vida del ser humano, deja huellas indelebles, usualmente negativas, tanto en el carácter como en el desarrollo futuro de la personalidad. Felizmente, en el caso de Pedro no fue así. La figura materna fue reemplazada por una tía soltera, Sebastiana, y la paterna por varios imagos positivos. El primero de ellos tuvo carácter virtual, el abuelo paterno, don Pedro Ortiz Montoya. Este, figura intelectual de la región y ejemplo moral de la localidad, había fundado la primera escuela de la provincia (aún antes de que lo hiciera el Estado), a la cual denominó "El Liceo del Progreso". En homenaje a su destacada labor en la educación regional, el Instituto Agropecuario de Celendín lleva su nombre como reconocimiento perpetuo. El abuelo, al fallecer, mantuvo vigente su presencia dejando en casa una herencia intelectual: una pequeña biblioteca en la cual destacaban libros de Física, Química y Matemáticas -los primeros amigos de Pedro- refugio constante de su soledad, y vía de transmisión informacional imaginada con su primera figura de identificación, un abuelo orientador, protector y silencioso.

Más adelante, el tío Francisco, alumno del abuelo, se convirtió en figura real de protección y en verdadero mecenas. No solo le enviaba libros actualizados desde Lima, sino que, con las mejores intenciones, ofreció pagarle los estudios universitarios cuando llegara el momento. Tal circunstancia nunca cristalizó, por el deceso del generoso tío. Mientras tanto, los libros, bien utilizados como armamento pesado, servían para que Pedro derrotara a sus ocasionales competidores por el primer puesto escolar, aparte de proporcionarle uno de sus adolescentes placeres favoritos: "plantear preguntas difíciles a los profesores para ponerlos en apuros".

En el curso de su vida escolar, el colegio constituyó para Pedro el espacio de la amistad y el compañerismo por antonomasia. Allí encontró otra figura orientadora, el profesor Don Eusebio Horna Torres, quien, aparte de enseñar matemáticas con didáctica de excelencia, constituía paradigma viviente de cómo ayudarse a sí mismo para triunfar. De extracción humilde, se había ganado la vida como policía. Con esfuerzo y gran perseverancia logró ingresar a la Universidad Católica, para, después, fiel a su terruño, volver a Cajamarca a enseñar a los chicos de su región. Tal profesor, no solo despertó el cariño y respeto de Pedro, sino que lo inició en lo que podría ahora considerarse como un modelo educativo integral para ciertas regiones del país: educar más allá de los linderos del colegio y conectar al estudiante, desde temprano, con su propia realidad socio-económica, cultural y ecológica. El Profesor Horna aplicaba técnicas innovadoras. Los alumnos, guiados por él, cultivaban, en un terreno aledaño, diversas legumbres que luego vendían en el mercado local. Organizó, además, una tienda escolar, cuyas ganancias contribuían al desarrollo del Colegio y obras de bien social. Publicaban un periódico que hacían circular por el pueblo incentivando a la población a tomar conciencia de los

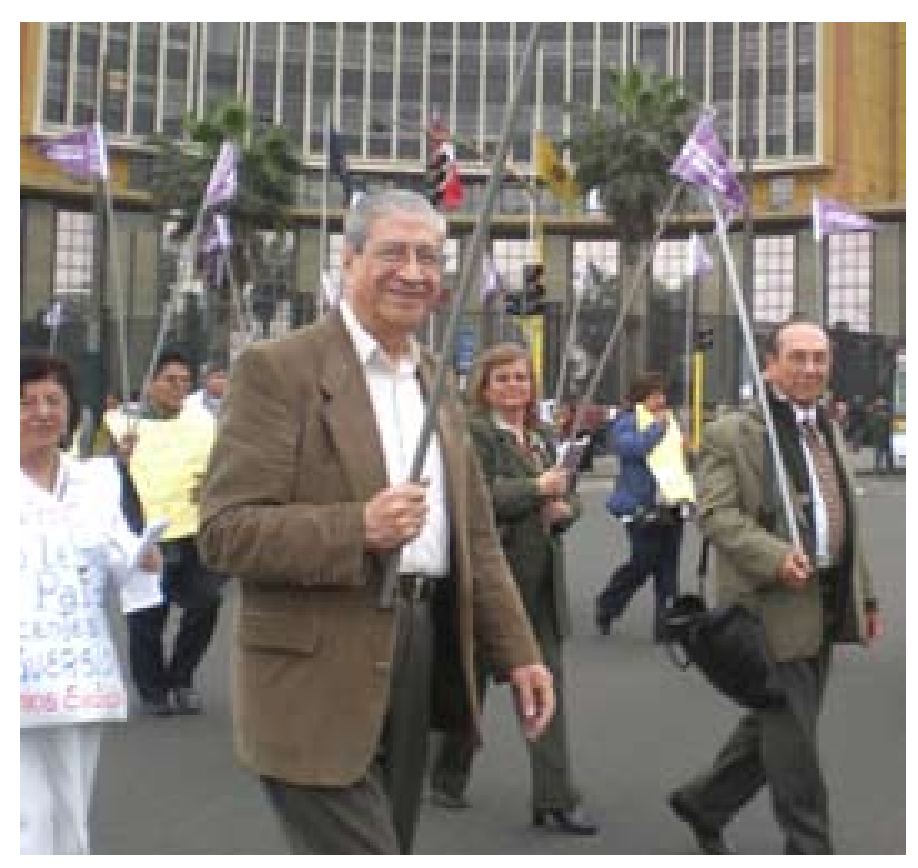

Figura 2: El Dr. Pedro Ortiz, en marcha de reclamo por la homologación docente. A su lado el Dr. Alberto Perales. 
problemas comunitarios, y demostraban su compromiso comunal dando conferencias de divulgación en diferentes distritos, en una suerte de campaña educativa rural.

Pero, el colegio también enseñó a Pedro los códigos morales de la buena conducta, y entre ellos, a respetar el valor del tiempo. Al respecto me contó la siguiente anécdota:

Pedro vivía a escasos metros del colegio y, por ello, solía llegar temprano a la escuela. Las $8.00 \mathrm{am}$, era la hora límite en que cerraban la puerta de ingreso. El alumno que llegaba tarde era objeto de medida disciplinaria: un joven fortachón de $5^{\circ}$ de Media, se encargaba de darle una buena reprimenda acompañada de unos cuantos palmazos. Si el estudiante se negaba a recibir tal castigo, obligaba a la aparición de otros dos, quienes, sujetándolo fuertemente, facilitaban la efectividad de la pena. Un día, Pedro se distrajo en casa $y$, aunque corriera apurado los escasos metros de distancia, llegó un minuto tarde. La puerta estaba cerrada. No hubo escapatoria y tuvo que pasar la prueba. "Desde entonces -me comentónunca he llegado tarde a otro compromiso. El colegio me enseñó el valor del tiempo y isabes? no entiendo por qué ahora, un simple castigo físico al alumno es considerado un delito..... Y, agregó, si le preguntas a un escolar qué tiempo le toma amarrarse los zapatos o lavarse y cambiarse de ropa, no sabe qué contestar; no conoce el valor del tiempo, porque no se lo han enseñado". Pedro sí sabía; por eso era motivo de admiración en la Facultad que, en un país dónde se ironiza 'la hora peruana', él fuera ejemplarmente puntual.

Terminada la secundaria, Pedro decidió ingresar a la universidad de Trujillo. Seguiría Ciencias. Había elegido Física como su futura carrera. Creyó que su destino estaba trazado. Sin embargo, no fue así. El mismo día en que debía matricularse, esperando en la fila de alumnos su turno para hacerlo, se encontró circunstancialmente con un tío, invidente y buen músico, al que hacía un buen tiempo no veía. En un breve diálogo Pedro le explicó su elección. El tío le preguntó con ingenua sencillez ¿Y cuánto ganan los físicos?... Pedro se matriculó en Medicina. Otra vez el destino lo enrumbó.

Ya en Lima, en la Facultad de San Fernando, Pedro se entusiasmó prontamente con una especialidad -la psiquiatría- a cuya lectura dedicaba todo su tiempo libre. Justo cuando pensó estar decidido a seguirla, el destino volvió a darle otro empujoncito desviándolo de su propósito original: un profesor amigo, el Dr. Carlos Mejía Bejarano, le comentó que estaban buscando un Jefe de Prácticas para el curso de Neuroanatomía, puerta de ingreso ineludible para la especialidad de Neurología. Pedro se hizo neurólogo. Es decir, llegó así a la carrera que mejor sustento científico ofrecía a su futuro trabajo intelectual y que habría de permitirle, llegando a las Neurociencias, elaborar una teoría socio-biológica-informacional del Hombre, que quizá, de otra manera, no le hubiera sido posible.

Posterior a su graduación, el resto del recorrido profesional de Pedro estuvo insistentemente plagado de éxitos que, anotamos sucintamente, tal como él mismo las jerarquizara al preguntarle cuáles consideraba sus mayores logros:

Haber cumplido treinta años de labor asistencial en los servicios médicos de la Seguridad Social, y desempeñado el cargo de Jefe de Servicio de Neurología, así como el de Jefe de Departamento de Neuropsiquiatría, del cual fue separado por razones políticas.

Haber cumplido más de 50 años en la docencia universitaria en la Universidad Nacional Mayor de San Marcos, en las Facultades de Medicina, Psicología y Educación; labor educativa que incluyó, además, actividades docentes en la Universidad Nacional Federico Villarreal, la Universidad Particular Inca Garcilaso de la Vega, la Universidad de Lima y la Universidad Femenina del Sagrado Corazón.

Dentro de la actividad docente sanmarquina, haber sido organizador y coordinador del Doctorado en Medicina, creador del Doctorado en Ciencias de la Salud; creador y coordinador de la Diplomatura, Maestría y Doctorado en Neurociencias; co-fundador y Director del Instituto de Ética en Salud de la Facultad de Medicina.

Haber contribuido al desarrollo de la institucionalidad democrática dentro del gremio médico, habiendo llegado a ser Presidente del Cuerpo Médico de su Hospital y Decano del Colegio Médico del Perú. En su primer cargo, propugnador de la racionalidad en el uso de los medicamentos. Durante su gestión en el Colegio Médico, y a iniciativa del Consejo Regional de Lima, se inició formalmente la docencia de la Ética en las Facultades de Medicina del país. Pedro, acorde con sus valores sociales, fue siempre ejemplo de conducta profesional, incluyendo los momentos de reivindicación docente.

Haber desarrollado una teoría sociobiológica-informacional del Hombre y de su desarrollo como personalidad moral, una de las pocas que se ha publicado en un país subdesarrollado.

\section{EL INSTITUTO DE ÉTICA EN SALUD Y EL ACTA DE COMPROMISO MORAL}

El 12 de noviembre de 2002, con Pedro y otros profesores sanmarquinos ${ }^{(*)}$ fundamos el Instituto de Ética en Salud de la Facultad de Medicina de la UNMSM. El objetivo principal era investigar los problemas éticos, tarea compleja, que enfrentaba el reto de convertir el problema metafísico en problema científi-

\footnotetext{
(*) Los otros miembros fundadores fueron los doctores S Zavala, A Mendoza, D Molero, J Alfaro, M Oliveros, C Sogi, E Bravo, R Morales, V Espinoza, F Rivera, y las Enfermeras M Arcaya, C del Carmen, T Espinoza, J Durand y M Infante.
} 
co. Kerlinger considera tal pretensión como imposible, por la imposibilidad de objetivar y, aún menos, mensurar las variables del fenómeno ético ${ }^{(1)}$. Creativamente, el Instituto postuló que los problemas morales no surgen en abstracto sino que se expresan en conductas humanas. La conducta humana, como tal, sí es pasible de ser estudiada como problema científico. Sobre este planteamiento básico, los miembros del instituto han realizado investigaciones y vienen investigando en el campo de la ética social, institucional e individual.

Para Pedro, su primer presidente, el Instituto fue arena de actividad académica y de polémicas éticas, filosóficas y metodológicas, así como simiente de amistad y compañerismo laboral.

Uno de sus aportes fue generar el Acta de Compromiso Moral para los docentes, discentes y administrativos de la Facultad de Medicina que, a diferencia de otros códigos deontológicos en que el compromiso del firmante es con instituciones, autoridades o deidades, en este, el compromiso es consigo mismo.

\section{SU ENFERMEDAD Y MUERTE.}

En marzo de 2010, Pedro acompañó a su señora, Luisa, a su consulta especializada. La médico -amiga y discípula- ordenó una batería de análisis sanguíneos, entre ellos, la determinación de marcadores biológicos oncológicos. Medio en broma, medio en serio, solicitó si también pudieran hacérselos a él, como seguimiento a una prostatectomía ya ejecutada. Así lo hicieron. Los análisis de su señora salieron normales mientras que los de Pedro fueron altamente positivos. No creyendo en los resultados, los confirmó en un laboratorio particular. Los títulos resultaron aún más elevados. La enfermedad lo golpeó justo en el momento en que su teoría salía del ámbito nacional. Había sido invitado a Paraguay para exponerla y hubo de cancelar su viaje. Comienza, entonces, la peregrinación por diversos consultorios y especialistas y es sujeto

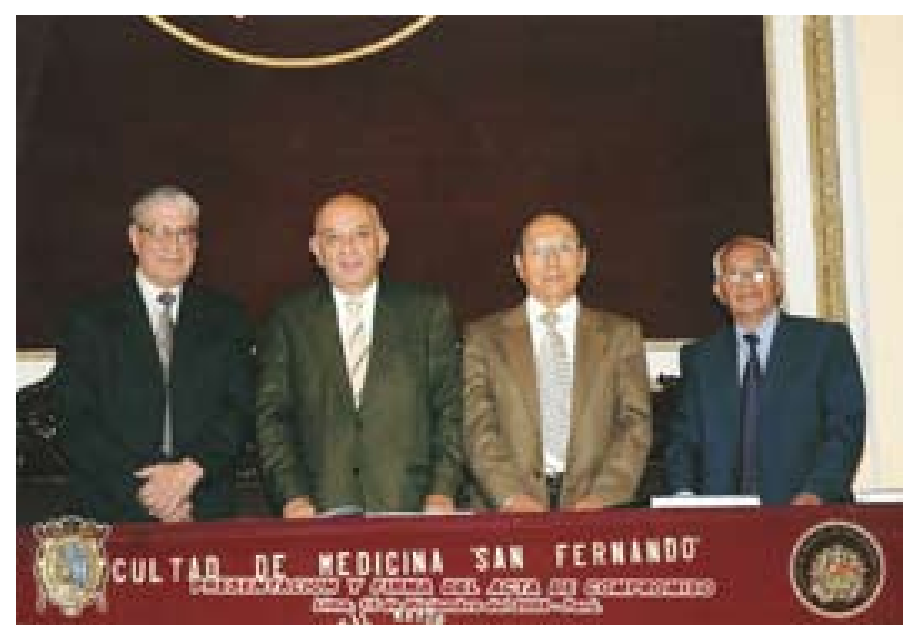

Figura 3. En la presentación y firma del Acta de Compromiso Moral. De izquierda a derecha: Dr. Ortiz, Dr. Emilio Blanco (Decano de la Facultad de Medicina), Dr. Alberto Perales (Director del Instituto de Ética en Salud) y Dr. Salomón Zavala.

de exámenes cada vez más sofisticados. Una tomografía computarizada helicoidal, una biopsia y una gammagrafía ósea confirman el temido diagnóstico a nivel pulmonar, en Grado IV, con expansión metastásica. Repetidos ingresos al hospital Rebagliati no pueden vencer la enfermedad.

Es justo destacar aquí, la excelente atención que Pedro recibiera de todo el personal hospitalario: autoridades, médicos, enfermeras, auxiliares y otros profesionales de la salud. Y más justo aún, relevar la altísima calidad científica y humana de sus dos médicos de cabecera, los Drs. Nahun Tamayo, internista, y Gabriela Ferrel, oncóloga.

A pesar de la gravedad de la situación, Pedro no pierde su buen humor y atiende con cordial trato a los innumerables amigos y discípulos que continuamente lo visitan. Soporta los efectos de la quimio y radioterapia, y el dolor físico del proceso, con valentía y ejemplar estoicismo. Hasta los últimos momentos en que su lúcida mente lo acompañara, su obsesión es escribir, plasmar sus conocimientos y las verdades que había descubierto en enseñanza permanente, su sublime obsesión por dar. Si algo caracterizó a Pedro fue su eros pedagógico, enseñar y dar al prójimo; vale decir, la impronta del verda- dero maestro, pues Maestro no es quien te instruye, sino aquel que te enseña a descubrir quién eres y cuánto puedes.

Los últimos meses, y particularmente días, su mayor sufrimiento no es somático sino espiritual, no poder terminar su obra y los libros que tenía en mente. Penosamente, una complicación neurológica termina por incapacitarlo, pero aún así persiste en el intento con ayuda de un Residente; pero, la enfermedad ya no se lo permite. Metástasis cerebrales causan síntomas neurológicos, aunque sin comprometer el sensorio ni las altas funciones intelectuales; el dolor se va pronunciando. Finalmente, es inevitable sedarlo a nivel de inconsciencia, para evitarle mayor sufrimiento. Pedro fallece el 21 de marzo de 2011, en el hospital Edgardo Rebagliati, rodeado del cariño de su esposa Luisa, tres hijos, Rosa, Pepe y Cecilia, su hermano Lolo y 5 nietos.

En sus exequias le tributan diferentes homenajes, entre ellos, uno organizado por el Colegio Médico. En este, el actual Director del Instituto de Ética en Salud, Dr. Salomón Zavala Sarrio, compendia su discurso: "Ha muerto un HOMBRE.........en esencia, un hombre BUENO......". Innumerables instituciones, alumnos, colegas, amigos y familiares le rinden sentido homenaje. 


\section{¿QUÉ SIGNIFICA PARA SAN MARCOS LA TEORÍA DE PEDRO ORTIZ?}

La teoría socio-biológica informacional del Hombre de Pedro Ortiz, se basa en una nueva tesis sobre la información que modifica sustancialmente los conceptos de vida, evolución, sociedad, conciencia y personalidad. Desde tal perspectiva, el modelo del sistema nervioso humano resulta esencialmente diferente al del animal. La teoría conceptúa la personalidad como el individuo transformado socialmente en ser moral y no como el conjunto de características o rasgos personales como solía considerarse.

Pedro postula que la especie Homo sapiens ha sufrido sucesivas transformaciones $y$, a diferencia de las especies animales que viven en manadas constituyendo grupos multi-individuales, ha sido capaz de formar grupos supraindividuales capaces de crear cultura (información social). En tal sentido, se ha convertido, primero, en Humanidad, y luego en Sociedad. El Hombre es, así, el resultado de la expresividad epigenética de su potencial hereditario influenciado y re-estructurado cinéticamente por la sociedad en la cual existe. La información social que la sociedad habrá de proveerle por medio de sus estructuras y de otros seres humanos, será codificada en su neo-córtex, estructurando gradualmente su conciencia y convirtiéndolo, progresivamente, en una personalidad de naturaleza moral. El hombre no tiene una personalidad sino que es una personalidad ${ }^{(2-6)}$.

La teoría ha generado desarrollos no solo en el campo de las neurociencias, la psicología y la psiquiatría, sino que posibilita replantear la naturaleza y objetivos de las ciencias médicas y de la salud, así como los de la educación y la ética como ciencias sociales. Por ejemplo, en su marco conceptual, la Ética puede definirse como la ciencia social que estudia los procesos de transformación de la Humanidad en una Sociedad estructurada moralmente; en tanto que, la Educación es la ciencia social que estudia los procesos de transformación del individuo en una Personalidad, que debiera ser íntegramente moral.

Los escenarios para el desarrollo de sus propuestas teóricas fueron tres $\mathrm{Fa}$ cultades sanmarquinas: la de Medicina, donde su preocupación principal fue explicar el desarrollo de la personalidad y moral del Hombre y sus trastornos por lesiones cerebrales; la de Psicología, donde el punto de partida fue cómo usar las ideas de Bernstein y Luria de los sistemas funcionales para explicar aquella relación y aplicarla en Psicología; y la de Educación, donde, una vez desarrollada la teoría, resultaba necesario introducir una explicación del desarrollo formativo de la conciencia y la personalidad, para que el profesor, conocedor del sujeto de educación, pueda influirlo con su información social.

El Hospital Rebagliati -como sede docente de pre y posgrado sanmarquino- y donde Pedro enseñó muchos de estos cursos, constituyó la base clínica-casuística que dio sustento práctico a sus especulaciones iniciales. De este modo, Pedro deja una teoría de la Personalidad que incluye una forma distinta de integrar sociedad-conciencia-personalidad, dando una solución distinta al problema mente/cerebro que las ciencias naturales no pudieron resolver. Esta teoría puede permitir, en el futuro, explicar la doble determinación patogenética y patocinética no solo de las enfermedades sino de la integridad del Hombre como persona.

No tengo duda alguna que la influencia de su teoría en la vida intelectual del Perú irá creciendo con el transcurrir de los años, en la medida que sea conocida. Ello dependerá no solo de la labor de difusión que habrán de desarrollar sus discípulos y amigos, sino de la toma de conciencia de las autoridades universitarias sobre la importancia de la misma.

Conviene considerar, sin embargo, que Pedro deja un legado heurístico que aún requiere de comprobación. Y que una manera realista de contribuir a perennizar su obra es someter al es- crutinio científico muchos de sus postulados, pues él mismo, de haber continuado su existencia, lo hubiera hecho. Solo así demostraremos la verdadera importancia de su legado. La UNMSM, como universidad pública y su alma mater, está más obligada que cualquier otra a ejecutar esta tarea. Debiera abrir una línea troncal de investigación sobre la teoría señalada que, alimentada por el talento de tantos jóvenes sanmarquinos, construya sobre su base, el edificio necesario de conocimientos para contribuir a la solución de los problemas nacionales.

La teoría abre enormes campos de estudio y aplicación correctiva a los programas educativos, basándolos, ya no en la exclusiva consideración intelectual del ser humano, sino en la totalidad de su ser, integral y moral, así como en los problemas sociales plagados de injusticia, violencia, corrupción y otras variables destructivas. De este modo, ofrece las bases para generar un plan que podría reducir la enorme distancia que media entre lo que somos y lo que debemos ser.

Veamos como resume Pedro el tema, con ocasión de su discurso de aceptación del Grado de Doctor Honoris Causa que le otorgara la Universidad Nacional de Educación Enrique Guzmán y Valle, el 29 de septiembre de 2010:

"En efecto, creo, en primer lugar, que una vez explicada la naturaleza informacional de los procesos esenciales que determinan la existencia de la vida, la sociedad y las personas, el cuidado del planeta, de los recursos naturales y de nuestra propia vida, debe ser responsabilidad social superior de los pueblos actualmente en situación de subdesarrollo. Porque es mi convicción que el hecho de tratarnos como seres apenas diferenciados de los primates es consecuencia de la teoría científica natural que, si bien sirve para el fomento de la tecnología y la industria, también sirve para justificar el egoísmo, la dominación y la injusticia.

En segundo lugar, creo que una vez explicado el estado actual de la humanidad, 
esta debe llegar a transformarse en una sociedad realmente solidaria, realmente libre y realmente justa. Porque es mi convicción que este es el papel de la teoría ética y de todas las estrategias de moralización social, entre las cuales, la educación juega el rol fundamental y más importante.

En tercer lugar, creo que una vez superados los problemas del dualismo mentecerebro y del reduccionismo cognitivista, fuese funcionalista o emergentista, todo niño que ingresa a una institución educativa debe formar y desarrollar su conciencia a plenitud, para que, de este modo, cada uno de ellos llegue a ser una personalidad realmente digna, realmente autónoma y realmente íntegra. Porque es mi convicción que toda institución educativa ya no debe reflejar el estado real y actual de la humanidad, sino que debe organizarse como un sistema relativamente aislado que realiza en toda su estructura y relaciones, el modelo de sociedad moral que aspiramos.

En cuarto lugar, creo que una vez planteado el problema de la relación entre la sociedad, la conciencia y la personalidad, la teoría sociobiológica que lo resuelve debe ser la base de una neurociencia social que explica la verdadera naturaleza de la actividad del cerebro de las personas. Porque es mi convicción que el modelo naturalista del cerebro del mamífero jamás podrá explicar la actividad consciente del cerebro del hombre, y que solo una neurociencia social puede servir para que todo maestro pueda conocer y comprender la historia de sus alumnos, a fin de aplicar la estrategia educativa que realmente necesita cada uno de ellos.

$Y$, en quinto lugar, creo que una vez definida la educación $1^{\circ}$ ) como la ciencia social que explica los procesos por los cuales cada ser humano que nace se transforma en una personalidad, únicamente dentro de una sociedad, y, 2ㅇ) como la tecnología social por medio de la cual se realiza dicha transformación dentro de una sociedad concreta, la sociedad en su conjunto debe asumir la responsabilidad total del destino de cada niño que se forma en su seno; $y$, por lo mismo, el educador que representa a esta sociedad debe asumir la máxima responsabilidad en la formación moral de los sentimientos, los conocimientos y las motivaciones de cada uno de sus alumnos, para garantizar, de este modo, que toda personalidad moralmente formada pueda contribuir como trabajador a la transformación igualmente moral de la sociedad".

La convicción de Pedro era que "una teoría del Hombre no solo debía explicar qué fue y qué es ahora, sino también lo que debe ser, hacer y saber, y, entre estos saberes, aquél que le recuerda que su porvenir como especie no se encuentra en otro planeta, sino en esta Tierra de cuya materia está hecho y a la cual debe volver". Porque creía que "una sociedad moralmente organizada es la única forma de garantizar las mejores condiciones del planeta para la prosecución de la vida bajo la absoluta responsabilidad de todos y cada uno de los hombres".

\section{PALABRAS FINALES}

El cuerpo docente, personal administrativo y alumnado de la Facultad de Medicina de San Fernando, y muy en particular, los miembros del Instituto de Ética en Salud, sufren su partida. Debemos agradecerle a Pedro no solo habernos regalado sus enseñanzas y sabiduría sino haber practicado la éti- ca con el ejemplo, a tal punto que, un hombre sencillo, intentando definirlo, lo hizo en los siguientes términos: "Don Pedro, es ejemplar de una especie ya en extinción..."

Por mi parte, a pesar de la tristeza que produce su desaparición, me queda un fondo de felicidad y agradecimiento al destino por haber compartido, como amigo y colega, con él y otros profesores sanmarquinos, no solo sabrosas discusiones científicas, filosóficas y culturales en el Instituto de Ética en Salud, sino también su existencia, una existencia plena de valores, de virtudes, de aspiraciones por un mundo más justo y moral, por un Perú mejor para los peruanos y una sociedad más humana y moralizada.

\section{REFERENCIAS}

1. Kerlinger F. Investigación del Comportamiento. Técnicas y Metodologia. Interamericana.México, 1985.

2. Ortiz P. La Formación de la Personalidad. Dimaso Editores, Lima, 1997

3. Ortiz P. Lenguaje y Habla Personal. Fondo editorial, UNMSM, Lima, 2002.

4. Ortiz P. Cuadernos de Psicobiología Social 6. E Nivel Consciente de la Actividad Personal. Fondo Editorial, UNMSM, Lima, 2004.

5. Ortiz P. Cuadernos de Psicobiología Social 1. Introducción a una Psicobiología del Hombre (Segunda Edición). Fondo Editorial, UNMSM, Lima, 2009.

6. Ortiz P. Educación y formación de la personalidad. Fondo Editorial UCH. Lima, 2008.

Agradecimientos:

A la Dra. Luisa Sakamoto Vda. de Ortiz, por la información ofrecida.

A la Profesora Martha Martina, por la fotografia facilitada.

Correspondencia:

Correo electrónico: perales.alberto@gmail.com 